\title{
LE BICENTENAIRE DE LA RÉVOLUTION
}

\author{
* \\ REVUE CRTIQUE \\ par \\ Dominique BOUREL
}

On a publié plusieurs milliers de livres en France et à l'étranger sur la Révolution française'. Cette commémoration a d'ailleurs été déjà beaucoup célébrée dans la presse spécialisée comme dans les médias et rien n'indique un tassement de cette logique des anniversaires tant cette fin $d u x{ }^{\prime} I^{\mathrm{e}}$ siècle fut chargée d'événements et de sens en Europe occidentale.

Nous voudrions revenir sur quelques publications marquantes, reçues à la Revue de synthèse, sans hésiter à trier ce qui fait progresser la recherche de ce qui ne devait être qu'une bonne operation éditoriale. De même, il n'est pas question de trancher en si peu de pages entre les interpretations divergentes polarisées autour des noms de François Furet et de Michel Vovelle, débat dans lequel les épigones et les journalistes ont souvent inutilement durci les clivages ${ }^{2}$. L'un des intérêts majeurs de cet anniversaire est d'avoir réouvert le dossier de l'historiographie de la Révolution, notamment avec La Légende de la Révolution ${ }^{3}$ ou la

1. "Les livres de la Révolution française", numéro hors série de Préfaces, mai 1989 et les deux volumes d'Alfred FIERRo, La Bibliographie de la Révolution française, Paris, Références, 1989. Des éditeurs et des institutions ont créé une centaine de collections : « $\mathbf{L a}$ librairie du Bicentenaire de la Révolution française " subventionnée par quatre ministères, la «Collection du Bicentenaire de la Révolution française " de l'Université de Franche-Comté à Besançon, «Ancien Régime, Aufklärung und Revolution », R. Oldenbourg Verlag (München), « Histoire provinciale de la Révolution française », Privat (Toulouse), etc. Rappelons, enfin, le régulier Bulletin d'histoire de la Révolution francaise, publié par la Commission d'histoire de la Révolution française du C.T.H.S. (le volume 1986-1989 est paru en 1990). Robert Maxwell a préparé une gigantesque réalisation multimédia avec des livres, des microfiches, des compacts et des vidéodisques, chez Pergamon Press.

2. Michel Vovelle, Les Aventures de la raison, entretiens avec Richard FiguIER, Paris, Belfond, 1989 ; Olivier BÉTOURnÉ, Aglaia Irmgard HARTG, Penser l'histoire de la Révolution. Deux siècles de passion française, Paris, La Découverte, 1989; François FureT, «Les mutations de l'historiographie révolutionnaire ", Bulletin de la Société française de philosophie, 3, 1989, p. 77-110; Claude LANGLors, "François Furet : l'atelier de la Révolution ", Esprit, juin 1990, p. 12-21.

3. Actes du colloque international de Clermont-Ferrand, 25-27 juin 1986, recueillis et présentés par Christian Croisille et Jean Ehrard, Clermont-Ferrand, Adosa, 1988. 
réédition très opportune d'Émile Ollivier, 1789 et 1889 : la Révolution et son ceuvre sociale, religieuse et politique ${ }^{4}$. Depuis Conscience religieuse en Révolution de Bernard Plongeron ${ }^{5}$, c'est l'un des nombreux mérites de François Furet et de son groupe de s'être penché sur cette question ${ }^{6}$. Cette rapide revue signalera d'abord quelques publications de documents puis quelques ouvrages dont on peut dire, sans beaucoup se tromper, qu'ils marqueront l'interprétation. On ne s'étonnera pas que la Revue de synthèse, dont toute l'existence fut un combat en faveur d'une histoire culturelle non isolée des paramètres économiques et sociaux, se réjouisse du retour à l'histoire des idées, politiques et philosophiques, un temps délaissée pour une approche plus matérielle parfois même chosifiante et déterministe de la réalité humaine.

Fidèle à sa tradition d'excellence, la "Bibliothèque de la Pléiade " offre le premier tome des Orateurs de la Révolution française dévolu aux Constituants? Une grosse introduction (CXXXII pages) situe les enjeux de l'édition, qui couvrira toute la période jusqu'en novembre 1799 , et en donne les clefs : ordre alphabétique des auteurs mais dans de grandes périodes chronologiques. Elle indique aussi les difficultés de l'établissement du texte. Un tableau thématique de notices et de notes (p. 1171-1598) est très copieux et contribue à faire de cette édition un outil de référence obligée et sûre. Parmi les multiples éditions des cahiers de doléances certaines ont été soignées comme les Procès-Verbaux et cahiers de doléances des sénéchaussées de Béziers et de Montpellier ${ }^{8}$. Mentionnons aussi l'intelligente anthologie présentee par Christine Fauré, Les Déclarations des droits de l'homme de 1789 : en effet, les divers projets - avec un bel inédit de Sieyès - constitue un véritable laboratoire des textes définitifs et on assiste ainsi au lent mûrissement des déclarations qui firent autorité $\dot{e}^{9}$. On doit à Jean Chaunu l'édition d'une série de textes afférents à l'histoire religieuse dont le bref Quod aliquantum. Avec la « constitution civile du clergé » et « l'exposition des principes sur la constitution civile du clergé », ils forment l'ossature de la politique religieuse du temps ${ }^{10}$. Enfin, Michael Walzer a eu l'excellente idée de donner le dossier de la mort du roi. Une controverse entre Ferenc Fehrer et

4. Introd. de Maurice AgulhoN, Paris, Aubier, 1989.

5. Sous-titre: Regards sur l'historiographie religieuse de la Révolution française, Paris, Picard, 1969. Pierre Colin, éd., Les Catholiques français et l'héritage de 1789. D'un centenaire à l'autre, Paris, Beauchesne, 1989.

6. Voir, par ex., Philippe Nieto, Le Centenaire de la révolution dauphinoise. Vizille, un mythe républicain, Grenoble, P.U.G., 1988.

7. Textes établis, présentés et annotés par François Furet et Ran HaLÉVI, Paris, Gallimard, 1989.

8. Collectés et présentés par Jean-Pierre Donnadieu, Montpellier, Archives départementales de l'Hérault, 1989. L'éditeur Denis Jeanson (Tours) a donné les Cahiers de doléances des six départements de la Région Centre. L'utilisation de l'ordinateur a considérablement aidé ce type d'entreprise : Robert JouvenOT, Le Bailliage de Baume-les-Dames en 1789. Les cahiers de doléances, Paris, Belles-Lettres, 1985 («Annales littéraires de l'université de Besançon ", 314).

9. Paris, Payot, 1988.

10. Droits de l'Église et droits de l'homme: Pie VT et les évêques français, le bref Quod Aliquantum et autres textes, éd. Jean Chaunu, Paris, Criterion, 1989. 
Walzer permet d'identifier la marque de ce crime dans la tradition politique ". Il apparaît donc clairement que, contrairement à ce qui fut écrit ici ou là, cette commémoration n'a pas à rougir de la comparaison avec la précédente en matière d'édition de documents. De plus, beaucoup de travaux auront une utilité, dépassant largement l'anniversaire, comme la bibliographie critique des journaux ${ }^{12}$ ou même le Dictionnaire des régicides ${ }^{13}$.

La différence entre les deux écoles historiographiques est évidente si l'on compare deux types de production alliant la positivité d'un savoir à sa réinsertion rationnelle et historique qu'on trouve dans les dictionnaires et encyclopédies. L'excellent État de la France pendant la Révolution (1789-1799) dirigé par Michel Vovelle ${ }^{14}$ présente une élégante synthèse à mi-chemin entre une nouvelle histoire de la Révolution et une encyclopédie; le pari engagé par ce dernier genre a été relevé et gagné par le Dictionnaire historique de la Révolution française, sous la direction d'Albert Soboul $(\dagger)$, mené à terme par Jean-René Suratteau et François Gendron ${ }^{15}$. Plus de mille entrees biographiques et thématiques amassent un énorme savoir. Bibliographies et renvois en font un remarquable instrument de travail. C'est une tout autre ambition qu'avaient François Furet et Mona Ozouf en réalisant le Dictionnaire critique de la Révolution française ${ }^{16}$. Il s'articule de façon critique en cinq chapitres : événements, acteurs, institutions et création, idées, interprétations et historiens. Les articles, qui sont plutôt des essais condensés, montrent l'émergence, la gestation et l'impact de l'idée démocratique dans la société française, ainsi que la profonde articulation entre 1789, 1793 et 1799 pourtant souvent distendue. C'est surtout par l'ampleur de la réflexion politique et philosophique que la lecture de ces pages s'impose, alors qu'une multitude de renvois facilite l'orientation. Le travail d'un événement dans une culture est admirablement disséqué et compris. Récusant les dogmatismes, ce dictionnaire préfère insister sur des problématiques soulevées par les différentes logiques à l'œuvre dans cette fin de siècle que sur des certitudes.

Cette tendance à l'aporie, ces méfiances devant les conclusions apparaissent aussi dans le fait que les livres les plus marquants sont souvent des actes de colloques. Des centaines furent organisés dans tous les points du globe et tous ne

11. Régicide et Révolution : le procès de Louis XVI, discours et controverse, trad. de l'anglais J. Debouzy et A. KuPIEC, Paris, Payot, 1989.

12. Pierre RÉtat, Les Journaux de 1789 : bibliographie critique, Paris, C.N.R.S., 1988.

13. Jacques-Philippe GıbouRY, Dictionnaire des régicides, 1793, Paris, Perrin, 1989. Roger Caratini, Dictionnaire des personnages de la Révolution, Paris, Le Pré-aux-Clercs, 1988.

14. Paris, La découverte, 1988.

15. Paris, P.U.F., 1989.

16. Paris, Flammarion, 1988. Rappelons aussi Alfred FIERRO, Jean-Francois FAYARD, Jean TUlARD, Histoire et dictionnaire de la Révolution française, Paris, R. Laffont, 1987, le superbe Dictionnaire Napoléon de ce dernier, Paris, Fayard, 1988, et la rééd. du célèbre Auguste KuscinsKI, Dictionnaire des conventionnels, 1916, Breuil-en-Vexin, Éd. du Vexin français, 1973. 
sont pas encore publiés! Un grand congrès mondial se tint en Sorbonne (612 juillet 1989) et portait sur l'image de la Révolution française. Cinq commissions, une masse imposante d'intervenants retiennent l'attention tant par les échos des recherches en cours que par les acquis, presque sans grand clivage ${ }^{17}$. Les trois volumes d'actes, disponibles dès l'ouverture, représentent une somme impressionnante de connaissances! Sous le titre général The French Revolution and the Creation of Modern Political Culture, trois volumes offrent les actes de colloques un peu plus restreints, quant à la participation, mais tout aussi globalisants et synthétiques, tenus respectivement à Chicago, Oxford et Paris entre 1986 et 1988. Ces tomes sont un modèle d'histoire culturelle très bien ciblée et problématisée, alliant la philosophie, la politique, la sociologie et d'autres disciplines auxiliaires ${ }^{18}$. Le premier volume ( 27 interventions) traite de l'Ancien Régime, ses représentations, son état et les formes de contestation politique. Une large partie est concentrée sur les concepts d'opinion publique, de représentation, de contrat, de citoyenneté et de souveraineté. Le second volume (vingt-trois contributions) insiste sur les contradictions et l'ambiguïté des conceptions politiques. Si la rupture est nette dès la convocation des États généraux, il faudra du temps aux acteurs pour mesurer la véritable signification non pas tant de leurs actes mais de leurs mots! L'idée de patrie et de régénération donne matière à de beaux développements. Le dernier volume ( 35 articles) étudie l'impact de l'événement et son devenir, à l'étranger comme dans la société française. Après avoir évoqué Burke - dont une nouvelle édition est désormais disponible ${ }^{19}$ l'interrogation se porte sur la Terreur, ses interprétations dans l'idéalisme allemand, ainsi que sur la contre-révolution ${ }^{20}$. Puis ce sont les rapports de la Révolution avec la religion - sur lesquels nous reviendrons - et, enfin, la Révolution lue à travers le prisme de ses plus grands interprètes, Quinet, Tocqueville, Marx, Michelet ${ }^{21}$. La sédimentation des commentaires se solidarise avec

17. M. Vovelle, éd., L'Image de la Révolution française, 3 vols, Oxford, Pergamon Press, 1989 ; un quatrième volume contenant les conférences plénières et les conclusions est sous presse.

18. Keith Baker, ed., The Political Culture of the Old Regime, Oxford, Pergamon Press, 1987; Colin LUCAS, ed., The Political Culture of the French Revolution, Oxford, Pergamon Press, 1988; François FURET, Mona Ozouf, eds, The Transformation of Political Culture 1789-1848, Oxford, Pergamon Press, 1989.

19. Edmund BURKE, Réflexions sur la Révolution de France, préf. Philippe RaYNaud, trad. de l'anglais Pierre ANDler, comm. Alfred Fierro, Georges Liebert, Paris, HachettePluriel, 1989; avec un choix de textes de Burke sur la Révolution, cette édition de P. Raynaud est remarquable.

20. L'excellent Gérard Gengembre, La Contre-révolution ou l'histoire désespérante, Paris, Imago, 1989; Matthias MIDDELL, "Konterrevolution während der französischen Revolution 1789 bis 1795. Zeitgenössischer Begriff und aktuelle Forschung ", ZfG, 1989, p. 97-114.

21. F. FURET, Marx et la Révolution franfaise, Paris, Flammarion, 1986 (avec les textes de Marx traduits par Lucien CALVÉ), et ID., La Gauche et la Révolution française au milieu du $X I X^{e}$ siècle, Paris, Hachette, 1986 (avec les textes de Quinet choisis par Marina VALENSISE). 
l'événement lui-même et lui restitue une cohérence. La trace de cette dernière fonctionne durant tout le $\mathrm{xIX}^{\mathrm{e}}$ siècle et le nôtre. Dans leur conception et leur qualité très sélective, ces trois volumes sont parmi les ouvrages les plus importants de cette commémoration. On ajoutera le beau recueil Die französische Revolution als Bruch des gesellschaftlichen Bewusstseins, colloque international tenu à Bielefeld ${ }^{22}$ en 1985, dans lequel on retiendra la place faite à la sémantique historique, la musique ${ }^{23}$ mais aussi la question de la rupture opérée dans le monde symbolique du XvIII siècle.

Outre les innombrables et souvent remarquables monographies locales, comme Les Débuts de la Révolution dans les pays de l'Ain 1787-179024, ou celles qui sont consacrées aux acteurs plus ou moins connus, tel celui qu'évoque le livre de Joël Cornette, Un Révolutionnaire ordinaire. Benoît Lacombe, négociant : 1759 $1819^{25}$, on peut souligner cinq domaines oủ la recherche est très active et a déjà tenu ses promesses :

- L'histoire des paysans, avec le colloque La Révolution française et le monde rural $^{26}$, la réédition très bienvenue des Questions agraires au temps de la Terreur de Georges Lefebvre ${ }^{27}$ et une belle monographie sur la Haute-Marne ${ }^{28}$, nous rappelle que la Révolution survient dans une France rurale à plus de $80 \%$ ! Ces paysans sont d'ailleurs souvent marqués par la piété et la religion dont on réexamine les rapports avec la Révolution.

- Un grand colloque dirigé par Bernard Plongeron fixe les acquis en la matière - de douloureuse mémoire - et indique de nouvelles pistes de recherche sans se limiter à la France et au catholicisme ${ }^{29}$. Plusieurs numéros de revue ont fait le point sur les rapports entre Église et Révolution ${ }^{30}$ et l'histoire des clercs a aussi retenu l'attention ${ }^{31}$. On peut dire que l'Église semble s'être réconciliée avec la Révolution ou du moins qu'un dialogue loyal s'est engagé entre les deux

22. Hg. von Reinhart Koselleck und Rolf ReichHard, München, Oldenbourg, 1988.

23. François Moureau, Elisabeth WAHL, Chants de la Révolution française, Paris, LGF, "Le Livre de poche ", 1989.

24. André Abbiatecl, Paul Perdrix, Bourg, Les amis des Archives de l'Ain, 1989.

25. Avant-propos d'Emmanuel LE Roy LAdURIE, Seyssel, Champ Vallon, 1986.

26. Actes du colloque tenu en Sorbonne les 23,24 et 25 oct. 1987, Paris, C.T.H.S., 1989.

27. Éd. revue et corrigé par Catherine KAWA, préf. de Guy LEMARCHAND, bibliogr. de Jean-Jacques CLĖRE, Paris, C.T.H.S., 1989.

28. J.-J. CLÈre, Les Paysans de la Haute-Marne et la Révolution francaise. Recherches sur les structures foncières de la communauté villageoise (1780-1825), préf. de M. VovelLE, Paris, C.T.H.S., 1988 ("Commission d'histoire de la Révolution française ", " Mémoires et documents ", XLIV).

29. Pratiques religieuses dans l'Europe révolutionnaire: mentalités et spiritualités, 17701820, Tumhout, Brépols, 1989. Rappelons Timothy TACKETT, La Révolution, l'Église, la France, Paris, Cerf, 1986.

30. Projet, 213, 1988 ; Comunio, XIV, 1989 ; Concilium, 221, 1989.

31. Rèed. de Bernard Plongeron, La Vie quotidienne du clergé français au xvif siècle, Paris, Hachette, 1988; Marie-Trinite KeRVINGANT, Des moniales face à la Révolution francaise, Paris, Beauchesne, 1989. 
protagonistes $^{32}$; il en va de même pour l'historiographie juive qui, longtemps unanime, est revenue à un jugement plus équilibré. Ici aussi des revues se sont fait 1'écho des débats du temps ${ }^{33}$ et Simon Schwarzfuchs a replacé dans le temps long l'émancipation avec $\mathrm{Du}$ juif à l'israélite ${ }^{34}$, alors que l'abbé Grégoire se voyait même édité deux fois ${ }^{35}$; il attend toujours son biographe ${ }^{36}$.

- L'histoire intellectuelle, late sensu, philosophique et politique fut au centre de superbes essais de Lucien Jaume ${ }^{37}$, Bronislaw Baczko ${ }^{38}$ et Marcel Gauchet ${ }^{39}$, qui, chacun, mériteraient une étude particulière. C'est aussi une excellente idée d'avoir édité des articles de Mona Ozouf sous le titre L'Homme régénéré ${ }^{40}$ dont l'intelligence et l'enthousiasme illuminent depuis tant d'années ce champ d'étude. Un colloque a détaillé également les rapports de la Révolution avec le droit, son histoire et sa philosophie et introduit une problématique technique mais capitale ${ }^{41}$.

- L'histoire du livre, discipline en pleine expansion, a permis à Robert Darnton et Daniel Roche de composer un catalogue qui est en même temps un beau livre, Revolution in Print ${ }^{42}$, alors que la Bibliothèque nationale de Paris consacrait une exposition sur « deux cents trésors entrés à la Bibliothèque nationale de 1789 à 1799 ", selon le sous-titre du catalogue ${ }^{43}$. On trouvera aussi dans les travaux de Roger Chartier et d'Henri-Jean Martin d'importantes contributions à notre sujet; ils sont trop connus pour être rappelés ici.

- Enfin, l'exportation des idéaux de la Révolution en Europe et dans le monde a donné lieu à une vaste production ${ }^{44}$. L'impact en Allemagne remplirait à lui seul une bibliothèque; il est un thème traditionnel dans lequel il est difficile

32. Paul Colin, éd., D'un centenaire à l'autre. Les catholiques francais et l'héritage de 1789, Paris, Beauchesne, 1989.

33. Yod, 27-28, 1988 ; Hamoré, 1989; Les Nouveaux cahiers, 97, 1989.

34. Du juif à l'israélite : histoire d'une mutation, 1770-1870. Paris, Fayard, 1989.

35. Henti Gregolre, Essai sur la régénération physique, morale et politique des juifs, Préf. Robert BADINTER, Paris, Stock, 1988 ; ID., ibid., préf. Rita Hermon-Belot, Paris, Flammarion, 1988. R. BADINIER, Libres et égaux... L'émancipation des juifs sous la Révolution française, 1789-1791, Paris, Fayard, 1989.

36. Voir l'épais dossier de B. PLONGERON, L'Abbé Grégoire ou l'Arche de la fraternité, Paris, Letouzey et Ané, 1989.

37. Le Discours jacobin et la démocratie, Paris, Fayard, 1989.

38. Comment sortir de la Terreur. Thermidor et la Révolution, Paris, Gallimard, 1989.

39. La Révolution des droits de l'homme, Paris, Gallimard, 1989.

40. Essais sur la Révolution francaise, Paris, Gallimard, 1989.

41. La Révolution et l'ordre juridique privé. Rationalité ou scandale? Actes du colloque d'Orléans, 11-13 sept. 1986, prés. M. Vovelle, t. I et II, Paris, P.U.F., 1988. Voir aussi Jacques BouINEAU, Les Toges du pouvoir ou la révolution du droit antique 1789-1799. Toulouse-Le Mirail, Eché, 1986 (cf. le compte rendu de Michel PERTUÉ, Revue de synthèse, 3-4, 1987, p. 518-521).

42. The Press in France 1775-1800, Berkeley/New York, University of California Press, 1989.

43. 1789. Le patrimoine libéré, Paris, Bibliothèque nationale, 1989.

44. Echanges internationaux idéologiques et culturels dans la mouvance de la Révolution francaise. Paris, Belles-Lettres, 1988 ; Région, Nation, Europe : unité et diversité des processus sociaux et culturels de la Révolution francaise, Paris, Belles-Lettres, 1988; Le Groupe de Coppet et la Révolution francaise, Lausanne/Paris, J. Touzot, 1988. 
de s'orienter ${ }^{45}$. La Révolution française et l'hellénisme moderne (38 interventions) est un magnifique exemple d'histoire comparée ${ }^{46}$. C'est toutes les politiques extérieures successives de la France qu'il faudrait envisager : Yves Lemoine a consacré un livre catalogue à La Diplomatie française pendant la Révolution qui ouvre comme il se doit de larges horizons et fait pressentir tout le matériel qui dort encore dans les archives ${ }^{47}$. La réédition, ou l'édition, de quelques rapports d'ambassadeurs et de consuls n'aurait pas été de trop pour suivre les canaux des résistances et des transmissions de la pensée révolutionnaire.

Il reste certes encore d'importantes divergences d'interprétation de la Révolution mais un large consensus semble régner sur le déroulement et les enjeux de l'événement, au moins dans ses phases initiale et terminale. Un flot de publications va continuer à apporter des documents nouveaux ou difficilement accessibles. La précision des enquêtes - comme l'Atlas de la Révolution française ${ }^{48}$ depuis 1987 - rendra bien des services. Il sera intéressant de voir si les changements intervenus - en hommage au Bicentenaire? - en Europe de l'Est trouveront un écho dans la production historiographique (pensons à celle venue de R.D.A qui n'existera sans doute plus lors de la publication de ces lignes). Il serait naturellement dommage que tout le travail souvent ingrat et tellement documenté, bien que parfois alourdi de scories idéologiques en provenance de ces pays, soit purement et simplement interrompu. Il demeure que nulle détermination économique et sociale ou même politique ne rendra jamais compte en totalité de la singulière irruption de cet événement fondateur dont la datation est ellemême déjà en soi un problème. Cette radicalité, cette rupture instauratrice, convoque toutes les disciplines, tous les efforts et toutes les intelligences pour la comprendre et la penser.

Dominique Bourel.

45. Gérald CHAIX, «L'année 89 ", Bulletin d'information de la Mission historique française en Allemagne, 19, 1989, p. 75-141; cf. infra p. 386-387, le compte rendu de J. LEFEBVRE, du livre Révolutions françaises et pensée allemande 1789-1871, prés. Lucien CALviÉ, Grenoble, Ellug, 1989.

46. Athènes, Librairie de lHestia, 1989.

47. Préf. de Roland Dumas, Paris, Michel de Maule, 1989.

48. Cf. le compte rendu de Jean-Yves GrENIER, infra p. 375-376. 
Atlas de la Révolution française. Sous la dir. de Serge BonIN et Claude LANGLoIS.

I : Routes et communications, dir. Guy ARBELlot et Bernard LePETT; II : L'Enseignement, 1760-1815, dir. Dominique JULIA, conception graphique Huguette Bertrand, Serge Bonin, Alexandra Laclau. Paris, Éd. de l'École des hautes études en sciences sociales, 1988 et $1987,20,8 \times 29,5,91$ p. et 105 p., cartes, graphiques, bibliogr.

Le Bicentenaire de la Révolution française a donné lieu à la publication d'une multitude de travaux de circonstances, souvent peu originaux. Il en va tout différemment avec la belle série des Atlas de la Révolution française, véritable événement dans la cartographie historique de la France. Deux traits majeurs la caractérisent. Le premier est son ampleur puisque, sous l'égide de l'École des hautes études en sciences sociales et de l'Institut d'histoire de la Révolution française, une vingtaine de fascicules sont prévus. Le second est la complexité de son ambition. Comment cartographier un phénomène d'abord politique, enserrer dans un réseau de graphiques, tableaux et cartes, un événement d'une durée relativement brève et que l'historiographie a surtout traité par les textes? Il existe certes des précédents illustres, comme l'analyse spatiale de la Grande Peur par Georges Lefebvre, mais trop ponctuels et peu nombreux pour réconcilier une tradition géographique de l'historiographie française et la Révolution. La multiplicité des approches et la grande diversité des thèmes abordés sont les conditions nécessaires mais exigeantes pour la réussite d'une telle entreprise. Les deux premiers volumes parus en sont une illustration.

Le premier consacré aux routes et communications contient quatre thèmes principaux : les voies, les hommes et les moyens d'action, les moyens de communication, la circulation. Ce simple énoncé situe bien la double perspective impliquée par ces atlas, due à la bivalence de la cartographie qui permet à la fois de constater et de saisir des évolutions. Ce premier volume contient, d'une part, un inventaire des réalisations de l'Ancien Régime, la carte des grandes voies de communication en 1789 (p. 15, par exemple), et un bilan des possibilités de transport alors disponibles, comme la série de cartes sur le service des voitures publiques (p. 44-51). D'autre part, il synthétise l'cuvre révolutionnaire par la représentation de l'extension des multiples réseaux (chap. 1) et celle de leur utilisation, de leur financement (de 1792 à la fin de l'Empire, p. 35-37) et des transformations de l'espace qu'elles impliquent.

La même dualité se rencontre dans le volume consacré à l'enseignement. Divisé également en quatre chapitres thématiques (alphabétisation et enseignement élémentaire, enseignement public, pensionnats, écoles techniques et universités), le souci d'une analyse de longue durée au sein de chacun d'entre eux est évident, ce que souligne D. Julia dans son introduction (p. 7) et comme le montre l'encadrement chronologique qui couvre la période de 1760 à 1815 , assez comparable à celui du premier volume. Le tableau qui est dressé de l'enseignement en France à la fin de l'Empire peut être utilement comparé à celui des années 1780 . Les profondes transformations législatives des années 1791-1793 dans ce domaine privilégié qu'est l'éducation sont ainsi mesurables dans leurs effets, comme en 
témoignent les deux belles cartes qui synthétisent l'évolution de l'enseignement public entre 1789 et 1812 (p. 30-31). Les destinées géographiques et temporelles des nombreuses innovations comme les écoles centrales (p. 40) ou les écoles techniques, autant que les transformations des institutions anciennes, des écoles élémentaires (p. 22) et des collèges (p. 30-31) jusqu'aux universités (p. 70-76), permettent comme dans le premier volume de dessiner l'évolution du paysage révolutionnaire.

Cet inventaire bien incomplet ne doit pas induire en erreur : la portée de ces atlas n'est pas seulement descriptive; ils suggèrent aussi nombre d'éléments explicatifs. Deux traits leur donnent cette dimension. Le premier est le souci de croiser un ensemble de données aux corrélations probables et éclairantes. Si les données sociales et scolaires sont le plus souvent mises en parallèle, des investigations plus larges ne sont pas exclues comme les séries de cartes qui confrontent population, taille des villes et densité des collèges (p. 37), scolarisation et économie en Champagne (p. 14), ou alphabétisation et industrialisation (p. 26). Le second est le recours fréquent à des échelles spatiales très variées qui permettent d'alterner études micro- et macro-géographiques. Dans le tome I, nombre d'analyses de réseaux ont une dimension locale comme les routes de l'Hérault en 1803 (p. 23) ou les canaux du Nord en 1789 (p. 30). Dans le tome II, plusieurs études fines sont menées grâce à l'emploi d'une échelle spécifique, la ville (Rouen, Reims, Amiens) pour croiser alphabetisation et richesse urbaine (p. 24-25) ou un ensemble plus vaste (Champagne, arrondissement de Lille, département de Haute-Garonne) pour saisir alphabétisation et scolarisation (p. 14-17). A ces deux dimensions rigoureuses de l'investigation, les auteurs ont su ajouter un souci de la diversité qui permet une prise en compte très élargie du sujet traité. Pour s'en tenir au premier volume, citons ainsi les nombreuses représentations graphiques des voyages ou missions effectués par des gens célebres (Young) ou des fonctionnaires plus anonymes (p. 59-68), le réseau des maisons de commerce (p. 13), la carte de la transmission des lois dans le district de Saint-Flour en 1791-1792 (p. 45), ou ce retour à l'événement avec la géographie comparée de la progression du roi et de ses poursuivants en 1791 et la carte de la diffusion de la nouvelle de sa fuite (p. 71).

Décrire cette masse d'informations dans son entier est exclu, d'autant qu'aux cartes et graphiques de synthèse s'ajoutent une liste détaillée des sources utilisées qui précisent la portée des données représentées, un ensemble de tableaux statistiques en fin de volume et une bibliographie générale. Si l'on precise, enfin, que chaque ensemble de cartes est décrit et soutenu par un texte concis mais dense, il est aisé de constater la richesse de cette publication, instrument de travail désormais indispensable à toute bibliothèque sur la Révolution française. 
Marie-Vic Ozouf-Marignier, La Formation des départements. La représentation du territoire français à la fin du XnII siècle. Préf. de Marcel Roncayolo. Paris, Ed. de l'École des hautes études en sciences sociales, 1989. $15 \times 22,5$, $363 \mathrm{p}$.

Au moment où l'on célèbre le Bicentenaire de la création des départements, le livre de Marie-Vic Ozouf-Marignier apporte un éclairage nouveau. Comme l'indique le titre de l'ouvrage, l'ambition de l'auteur ne consiste ni à tomber dans le travers monographique ni à présenter une histoire exhaustive de l'événement (encore que ses développements s'appuient sur une recherche documentaire importante) mais à dégager la représentation, peut-être vaudrait-il mieux dire les représentations, du territoire français qui se révèlent à cette occasion.

A cette fin, lauteur présente deux séries de point de vue : ceux qui se dégagent à la lecture des comptes rendus des travaux de l'Assemblée et du comité de constitution, d'une part, et ceux que manifestent les très nombreuses adresses envoyées par les notables locaux à l'Assemblée, d'autre part. L'idéal géométrique et rationnel des constituants, soucieux d'inscrire les principes révolutionnaires d'égalité et d'unité dans la nouvelle carte administrative du territoire, s'oppose aux réactions des notables locaux qui visent surtout, quelle que soit l'argumentation présentée, à conserver les avantages acquis sous l'Ancien Régime, voire à en conquérir d'autres.

En ce qui concerne les discussions qui se déroulent au sein de l'Assemblée, où s'affrontent notamment provincialistes et départementalistes, Marie-Vic OzoufMarignier met en lumière un élément important en abordant la question tant controversée de l'aspect centralisateur ou décentralisateur de la réforme. Si elle se garde en effet d'apporter une réponse univoque sur ce sujet, elle montre en revanche, d'une façon convaincante, l'émergence du postulat déterministe qui unit les constituants au-delà de leurs divergences : la conviction qu'en agissant sur l'espace de manière adéquate, on peut obtenir l'organisation politique idéale, pour reprendre ses propres termes. C'est pourquoi, au fur et à mesure que les travaux se déroulent à l'Assemblée, la discussion se concentre sur le choix du nombre de circonscriptions. A la faveur de ce glissement, « il n'est plus paradoxal d'asseoir une république une et indivisible sur un territoire divisé, de faire de la division elle-même le fondement de l'union ", conclut l'auteur qui exprime ici le véritable enjeu des débats et la rupture avec l'Ancien Régime.

Ce postulat déterministe est un excellent guide dans le maquis de la correspondance locale. Mais là, en un sens, les choses sont plus simples, puisqu'il ne sert pas tant à masquer des divergences idéologiques qu'à étayer un discours revendicatif. L'utilisation du modèle géométrique prôné par le comité de constitution pour déterminer un chef-lieu de département ou de district en fournit un exemple. Suivant la position d'une localité sur un territoire, le modèle est tantôt respecté, tantôt transgressé. Le centre se définit par rapport à un carré $-\infty$ qui est conforme au projet initial du comité - ou bien par rapport à un cercle, un point sur le cercle, voire une position excentrique par rapport à celui-ci. Oubliant les contraintes de la géométrie, les villes s'appuient tour à tour sur des arguments 
historiques, naturalistes, administratifs ou économiques pour se tailler une zone d'influence à la mesure de l'image qu'elles ont d'elles-mêmes.

Une typologie contrastée du réseau urbain se dégage de ces plaidoyers toujours convaincus mais où se glisse parfois une certaine dose de mauvaise foi. Les villes de montagnes s'opposent aux villes de plaines, les villes administratives aux villes commerçantes et parmi ces dernières celles qui ont un commerce de proximité à celles qui commercent avec des contrées éloignées. Si une vision patrimoniale et une volonté protectionniste de repli sur soi, héritées de l'Ancien Régime, se manifestent fréquemment, le souci des communications et de la complémentarité des terroirs apparaît également. Ainsi - et c'est un autre paradoxe de la réforme - le projet de division du territoire révèle l'importance acquise par les centres urbains en tant que pôles d'organisation, alors que les débats à l'Assemblée avaient souvent mis en lumière une image négative des villes, forgée à la fois dans les thèses physiocratiques et dans la représentation nouvelle du territoire conçu comme une simple surface pourvue d'une unité.

Absorbé tout à tour dans la lecture des débats idéologiques de l'Assemblée puis dans celle des revendications locales, séduit par le talent avec lequel Marie-Vic Ozouf-Marignier discerne dans ces discours les lignes de force d'une vision géographique du territoire en gestation, le lecteur aimerait que les différents protagonistes de cette histoire se mettent à échanger leurs arguments. Ce pourrait être l'objet d'un prochain ouvrage où les membres du comité de constitution et les notables locaux dialogueraient ensemble après avoir, dans le premier, discuté chacun de leur côté du bien-fondé de leurs démonstrations respectives.

Nicolas QUILLET.

Marie-Joseph ChÉnier, Littérature française. Préf. de Denis Woronoff, prés. et notes de Jean-Claude BonNet et Pierre Frantz. Paris, Belin, 1989. $13,5 \times 21,5,317$ p., index (« Rapports à l'Empereur sur le progrès des sciences, des lettres et des arts depuis $1789 »$, III).

Voici, par l'effet du Bicentenaire, une réédition opportune. Le Tableau historique de l'état et des progrès de la littérature française depuis 1789 répondait à une commande officielle : Marie-Joseph Chénier fit de ce chant du cygne (rédigé entre 1808 et 1811 , laissé inachevé, publié seulement en 1815 par une ironie de l'Histoire) un bilan justificatif en conformité avec ses convictions d'idéologue. Non, les Lumières et la Révolution n'ont pas provoqué la décadence de notre littérature : "La France agrandie n'est pas devenue stérile en talens " (p. 53). Telle est la thèse à laquelle, sans qu'il en ait conscience, l'auteur apporte un démenti constant à la fois parce qu'il s'impose silence sur les œuvres écrites sous la Révolution - une espèce de pudeur l'y contraint - et parce qu'il se réfugie dans un passéisme ruineux qui le rend opaque à tout renouveau : Chateaubriand, le Romantisme naissant lui demeurent étrangers.

Les trois textes liminaires sont également remarquables. D. Woronoff rappelle 
les circonstances dans lesquelles furent conçus les cinq Rapports à l'Empereur. J.-C. Bonnet étudie " M.-J. Chénier et l'Histoire littéraire » et fait excellemment la part des mérites et des limites de son ouvrage. P. Frantz analyse en fin connaisseur " Chénier juge du théâtre de son temps ".

Édouard GurTron.

Georges Cuvier, Chimie et sciences de la nature. Préf. de Denis Woronoff, prés. et notes sous la dir. d'Yves LaIssus. Paris, Belin, 1989. 13,5 × 21,5, 333 p., index (" Rapports à l'Empereur sur le progrès des sciences, des lettres et des arts depuis $1789 »$, II).

Dans la première partie de ce Rapport, Cuvier présente l'état de la chimie au début du XIX ${ }^{e}$ siècle. N'étant pas spécialiste de chimie, il avait sollicité le concours de chimistes français, tels que Guyton, Chaptal, Vauquelin, Berthollet et Fourcroy. Il divise cette première partie en trois subdivisions en se basant sur sa vision de l'attraction moléculaire. Malgré l'indication " depuis 1789 » figurant dans le titre, l'auteur doit remonter jusqu'au $X^{\prime} I^{e}$ siècle afin de retracer l'histoire de la théorie de la combustion, dont Lavoisier avait d'ailleurs établi la théorie exacte vers 1777 . Cet ouvrage ne comporte aucune mention de la théorie atomique. En effet, il fut présenté à l'Empereur en 1808 quelques mois avant la publication de l'ouvrage de Dalton. Enfin, ce Rapport comporte de nombreuses références à des articles et des ouvrages divers, ce qui en fait un outil de travail précieux pour ceux qui s'intéressent aux recherches historiques.

Akira YoshidA.

Joachim FisCHER, Napoléon und die Naturwissenschaften. Stuttgart, F. Steiner Verlag, 1988. 15,5 × 22,8, 390 p., index ("Boethius ", Bd 16).

Toute une littérature hagiographique présente l'empereur à la fois comme "protecteur» des sciences et comme amateur bien informé (voire plus...) en mathématiques et dans les sciences de la nature. Au-delà de l'hagiographie, il y a là une vraie question, puisque l'époque du Consulat et de l'Empire constitue, à la suite de la Révolution, un moment clef de la mise en place des institutions scientifiques en France, comme vient de le rappeler le livre de N. et J. Dhombres (Naissance d'un nouveau pouvoir : sciences et savants en France, 1793-1824). C'est pourquoi le travail méticuleux de Joachim Fischer vient à son heure. Refus de la légende, critique exhaustive des documents, inventaire minutieux y compris de 
nos ignorances (impossible de remonter avant 1912, pour assigner une source qui désigne de ce nom le "problème de Napoléon » - sur ce point, l'auteur reconnaît que l'état de la recherche demeure à peu près ce qu'il était en 1949) : tout le matériel est là, ordonné selon le "fil d'Ariane de la chronologie ", pour évaluer à la fois ce que savait et pouvait avoir lu Napoléon, quelles relations il entretenait avec les savants, quel rôle il a joué à l'égard des institutions.

On appréciera les pages consacrées à l'École polytechnique ou les exemples parfois dramatiques illustrant le rôle des problèmes financiers dans l'infrastructure scientifique. Mais le plus intéressant tient peut-être à la diversité des relations avec quelques grandes figures. D'un côté, la fidélité d'un Monge, prêt à accompagner le souverain déchu dans un possible exil aux États-Unis; d'un autre, l'étrange chassé-croisé qui voit le général Bonaparte succéder à Lazare Carnot chassé de l'Institut en même temps que du Directoire (le récit de l'élection, comme l'analyse des relations du Premier Consul avec l'Institut sont un des moments forts du livre), puis le même Carnot, devenu ministre des Cent-Jours, écrire au président de la Classe de mathématiques pour demander une élection sur le même siège car " l'Empereur a reconnu l'inconvénient qu'il y a de laisser vacante, dans la section de mécanique... la place que sa Majesté est obligée de laisser inactive de fait " (p. 270) ; enfin, le rôle des discussions personnelles dans la mise à jour de l'information scientifique de l'homme d'État ("viva voce Darstellung durch den Fachmann" - Monge encore, ou Berthollet, par exemple). Les relations avec Volta font l'objet d'un chapitre entier.

Volontairement, l'ouvrage ne propose pas de thèse générale (si ce n'est la méfiance devant une image de propagande que Napoléon fut le premier à construire) : il s'en tient à l'établissement du plus grand nombre de faits possibles, ce qui, dans un domaine aussi obscurci par les évaluations rapides, est un grand mérite.

Pierre-François Moreau.

Louis Pérouas, Grignion de Monfort et la Vendée. Paris, Cerf, 1989. 12,5 × 19,5, 129 p. (« Histoire »).

Comprendre une tenace légende : tel est l'objet de ce petit livre. Selon la tradition, le terrible soulèvement vendéen de mars 1793 serait l'œuvre - à retardement - de la prédication de Grignion de Monfort au début du XVIII siècle et des congrégations qui lui ont assuré une fragile pérennité ("mulotins" et «filles de la sagesse »). Le saint de la Vendée serait donc aussi le "père " de la Vendée, combattante et militante. L'enquête sur l'influence des disciples de Grignion de Monfort dans les soulèvements populaires de 1793 se révèle plus que décevante. Les " mulotins " n'ont pris aucune part aux opérations militaires; les sœurs ont soigné les Blancs comme les Bleus et, dans plusieurs cas, protégé des républicains au risque d'être maltraitées par les insurgés... La conclusion de Louis 
Pérouas est formelle : « on peut estimer la participation des disciples de Monfort à la guerre de 1793 comme une question nulle et non avenue " (p. 110). Dès lors, l'auteur s'attache à cerner l'origine de la légende. La première mise en cause des monfortins est le fait des émissaires parisiens, envoyés à l'automne 1791 pour surveiller les populations lors du remplacement des curés réfractaires et limiter les premiers troubles. Dumouriez, marechal de camp, dresse un tableau optimiste de la situation: "plus de bruit qu'il n'y a de danger ", mais note cependant : " les missionnaires de Saint-Laurent sont dangereux... Les sœurs de la sagesse, tout utiles qu'elles soient dans les hôpitaux, sont dangereuses et il serait bon de détruire leur chef-lieu de Saint-Laurent... Les missionnaires sont très dangereux ; il faut les séculariser et les disperser » (p. 111). Sans autre forme de proces, sans livrer des informations complémentaires, sans avancer l'ombre d'une preuve, les successeurs de Dumouriez entonnent le même refrain. Pour Louis Pérouas, "l'imaginaire des Bleus" fait de Grignion de Monfort le père de l'insurrection vendéenne. La violence des combats transformera en "hostilité durable " ce qui « aurait pu n'être qu'un fantasme passager » (p. 118).

Le devenir de la légende survit à l'épisode révolutionnaire car, paradoxalement, la restauration s'appuie sur cette même rumeur pour exalter, en sens inverse, la résistance vendéenne à l'œuvre déchristianisatrice de la Révolution... Les années 1880 voient une résurgence et une amplification de l'identification entre Grignion de Monfort et la Vendée. On préparait alors sa béatification (janvier 1888) et le centenaire de 1789 se profilait à l'horizon. Chacun fourbissait ses armes et affûtait ses ancétres... Républicaine depuis 1875, la France, par les lois de 1880-1882 sur les congrégations, avait ravive les peurs de l'Ouest et suscité une vague de nostalgie.

Au-delà de cette encombrante filiation, comment peut-on envisager la relation du missionnaire et du territoire de son action? Il faut réfuter l'idée qui vient trop facilement sous la plume des hagiographes selon laquelle l'Ouest était déchristianisé avant l'arrivée du prédicateur. Lorsque Grignion de Monfort pénètre en BasPoitou l'effort d'encadrement tridentin commence à porter ses fruits. Mesurer la ferveur religieuse d'un diocèse n'est pas aisé, mais des indices convergents permettent de saisir les diversités de ce «territoire biscornu». Vers 1710, le calvinisme, officiellement supprimé, reste important sur les hauteurs bocagères méridionales du diocèse de La Rochelle. Au nord, s'implantent en plus grand nombre les confréries du rosaire, tandis que le nord-ouest, qui sera effectivement le coeur de la Vendée militaire, fournit un nombre important de prêtres et de séminaristes... Mais, là, encore, il ne faut pas céder à un déterminisme a posteriori : la Bretagne voisine dépasse de très loin les records vendéens. L'action de Monfort se situe dans un contexte moyen. L'originalité de ses missions consiste à tenter de rejoindre pratique religieuse et sociabilité populaire, « au lieu des chansons, des contes et des jeux, il place le rosaire " (p. 46). Les cantiques qu'il compose s'inscrivent déliberément sur des airs profanes bien connus du peuple. Louis Pérouas est manifestement séduit par cet effort "d'équilibre " mais il souligne les aspects obscurantistes qui amènent Grignion à dénoncer tous ceux qui cherchent à "harmoniser la science et la foi " (p. 50). Le succès de Grignion demeure toujours un peu suspect, en marge de l'Église officielle, inquiète de ses 
excès, de ses initiatives grandioses et de la relative liberté qu'il donne aux laïcs. Pour des raisons semblables, le culte qui se développe sur son tombeau est tout juste toléré. Par contre, au fil du siècle, ses disciples se glissent de plus en plus aisément dans le moule de l'Église dominante. Alors que le saint était resté très modéré dans la critique du jansénisme, les "mulotins" participent violemment au combat contre les appelants et transforment certaines de leurs missions en chasse aux jansénistes. Ils adoptent la dévotion au Sacré-Cour inspirée par Marie Alacoque et relayée par les Jésuites.

Cette sensibilité religieuse de plus en plus nettement anti-gallicane ne pouvait s'entendre avec les partisans des Lumières. A ce titre, Louis Pérouas admet une " influence en pointillé »...

Monique Cottret.

Pratiques religieuses, mentalités et spiritualités dans l'Europe révolutionnaire (1770-1820). Actes du colloque Bicentenaire de la Révolution, Chantilly, 2729 nov. 1986, réunis par Paule Lerou et Raymond DARTEVELle, sous la dir. de Bemard Plongeron. Paris, Brepols, 1988. $17 \times$ 23,7, 777 p., cartes, graph., ill. ("Greco n ${ }^{\circ}$ 2, C.N.R.S. »).

Nous avons là les actes d'une table ronde du C.N.R.S. tenue à Chantilly en 1986, en préliminaire aux grandes manifestations du Bicentenaire. Ce colloque exemplaire avait réuni 250 participants dont 67 rapporteurs et communicants, dans une ambiance oủ les passions politiques ne débordaient pas encore l'intérêt scientifique. Toutes les sensibilités sont en effet représentées parmi les auteurs. A distance, ceci ressemble à un tour de force, une gageure réussie, peut-être l'une des rares dans la production scientifique de cette commémoration. Dans des discussions passionnantes et la présentation de points de vue nouveaux, l'histoire religieuse a gagné en précision, en profondeur problématique et en perspectives nouvelles.

Le but de la rencontre était ambitieux : tenter d'appréhender le comportement des croyants clercs et laïques, toutes confessions confondues, face aux événements révolutionnaires dans l'ensemble de l'Europe. Le travail a été organisé autour de huit thèmes, destinés à cerner le vécu religieux des populations, tout en privitégiant les sources documentaires mal connues ou mal utilisées. Bref, selon une attitude assez originale concernant la Révolution, dresser le bilan de l'enquête sur le terrain avant de penser la Révolution religieuse. Derrière ce travail, quelques Bastilles étaient tout de mème visées : briser le manichéisme entre bonne et mauvaise religion, étendre l'enquête aux pays conquis, étudier les phénomènes dans la longue duree, le temps d'une génération, entre 1770 et 1820 .

Il est hors de question d'aborder ici l'ensemble fort riche et dense des travaux de ce colloque. Une description des grands thèmes permet de s'en faire une idée. Un rapport de Gabriele de Rosa, enrichi de onze communications couvrant 
l'ensemble des pays concernés a d'abord étudié les « Mutations révolutionnaires et traditions confessionnelles dans les pays occupés ». Jean de Viguerie et onze communicants ont traité " $\mathrm{La}$ vie religieuse et la sacramentalisation des fidèles". Gérard Cholvy et dix communicants se sont attachés aux « Résistances et mutations culturelles ». Claude Langlois et six communicants ont décrit « La religion révolutionnaire ", tandis que Raymond Dartevelle et six autres s'attachaient à " La catéchèse et l'enseignement "; Louis Châtellier et cinq communicants à "Vie associative et confréries"; François Lebrun et sépt communicants aux " Dévotions et pèlerinages "; Freddy Raphael et six communicants à l'« Iconographie juive et chrétienne ", enfin.

Il faut souligner que les éditeurs de ce colloque ont pu publier les discussions riches et plurielles qui avaient tenu en haleine les participants. Le lecteur y trouvera des aperçus méthodologiques nouveaux (sur la typologie de la rupture, le syncrétisme entre culte de la Raison et culte de l'être suprême...) et de nouvelles voies d'interprétation (sur le dimorphisme sexuel, la mort et le suicide, le conformisme...) des sources nouvelles enfin (catéchismes...). Chemin faisant, on confirme l'importance de la Terreur dictatoriale (bien plus redoutable que son homologue montagnarde), le rôle des religieux et surtout des religieuses, celui des femmes enfin, lancées à corps perdu dans le catéchisme, la prédication, les messes sèches, les assemblées de prière.

Ce volume est heureusement complété par le catalogue de l'exposition sur "L'espace et le sacré à l'époque révolutionnaire » (dont une partie de la figuration est reproduite en couleurs). En conformité avec l'esprit de la formation du C.N.R.S. responsable du colloque (le G.R.E.C.O. $n^{\circ}{ }^{2}$ ), un recensement sommaire des sources manuscrites et des instruments de travail sont fournis aux chercheurs non spécialisés. Une chronologie des événements de l'histoire religieuse permet, en outre, de manipuler sans incertitudes le double comput de cette période. Un index des noms de personnes et des noms de lieux complète cet ouvrage, indispensable à tous ceux qui veulent penser la Révolution en refusant d'en rester paresseusement aux mythes habituels.

Nicole LEMAîTRE.

"Influences de la Révolution. Les sociétés musulmanes, le Japon, l'Amérique latine ", Revue internationale des sciences sociales, 119, 1989.

A l'occasion du Bicentenaire de la Révolution française, la Revue internationale des sciences sociales a consacré un numéro à l'étude des influences exercées par les idéaux de cette Révolution sur un certain nombre de pays extra-européens : les pays musulmans, le Japon, l'Amérique latine. Le choix de ce thème, doté par luimême d'un grand intérêt, présente encore l'avantage de mettre l'accent sur un aspect auquel les nombreux colloques et célébrations du Bicentenaire n'ont pas peut-être accordé toute l'importance requise. Pourtant, comme le rappellent les 
éditeurs, «la Révolution française a exercé son influence partout $[. .$.$] , elle a$ d'emblée revêtu un caractère universel et a diffusé son modèle ". Il convient donc de saluer doublement l'initiative de la Revue internationale des sciences sociales, qui nous présente six articles sur ce sujet, tous rédigés par des spécialistes avertis et soucieux de l'évolution spécifique des sociétés où ils cherchent à déceler l'impact de la Révolution française.

Les trois premiers articles sont consacrés aux rapports entre la Révolution française et les sociétés musulmanes.

Le premier est de Bertrand Badie, intitulé " L'impact de la Révolution française sur les sociétés musulmanes : évidences et ambiguïtés " (p. 7-18). Il s'agit d'un texte se situant à un niveau relativement théorique et assumant, de ce fait, une fonction introductive par rapport aux articles suivants. L'auteur, en effet, se référant à la totalité du monde musulman, cherche à cerner essentiellement les difficultés que rencontrent les sociétés musulmanes dans leur effort pour maitriser les idéaux révolutionnaires et analyse les tensions conceptuelles qui en dérivent. Il arrive ainsi à montrer que « l'impact de la Révolution française donne naissance à des synthèses pour le moins ambiguës, chacune exprimant un bricolage plus ou moins fragile d'éléments culturels endogènes et d'éléments importés » (p. 16). Cette démarche théorique, où la sociologie est constamment éclairée par l'histoire, lui permet ainsi de mieux déceler «le dilemme de l'impact de la Révolution française : ou il est sélectif et retraduit, et il perd alors l'essentiel de sa signification d'origine, se limitant au plus à susciter des innovations qui s'agrègent alors à de tout autres contextes; ou il est plus central et davantage approfondi, pour risquer alors de se briser dans un processus rapide de délégitimation " (p. 17).

Le deuxième article, qui a pour auteur Şerif Mardin, est intitulé : « L'influence de la Révolution française sur l'Empire ottoman » (p. 19-34). Malgré son titre, la moitié de cet article est consacrée à l'étude des rapports entre l'Occident et l'Empire ottoman avant la Révolution française, ce qui ne laisse pas d'introduire un déséquilibre assez gênant. Cependant, ce procédé permet de montrer que les célèbres réformes du Tanzimat, au XIX siècle, sont "l'aboutissement d'un lent processus de maturation" (p. 22). L'empreinte des idées de la Révolution française est décelable aussi bien à travers les nouveaux textes constitutionnels que s'octroie l'Empire ottoman que dans l'idéologie qui anime " une nouvelle race : les intellectuels réformistes " (p. 30). L'auteur, notons-le, souligne à juste titre la " tonalité islamique » de cette idéologie où s'opère une "synthèse étroite " (p. 32) entre les éléments de la culture française révolutionnaire et ceux de la culture ottomane.

Enfin, dans le troisième article, "La Révolution française et le monde arabe " (p. 35-46), Elbaki Hermassi s'intéresse essentiellement aux cas de l'Égypte et de la Tunisie. Là encore, nous relevons l'existence de mouvements de réforme importants (sous l'égide de Mohammed 'Ali, en Égypte, et sous celle d'Ahmed Bey, en Tunisie), bientôt suivis par l'émergence d'une génération d'intellectuels, qui "vont réfléchir sur la condition des sociétés islamiques et arabes, sur la grandeur et la décadence des civilisations et sur les conditions de la Renaissance " (p. 37). Parmi eux, se détachent les figures imposantes de l'Égyptien Tahtawi, auteur de la première description de la civilisation occidentale présentée aux 
lecteurs arabes, et du Tunisien Kheireddine, écrivain et homme d'État catégoriquement attaché, lui aussi, aux idéaux européens. Le rayonnement de ces idéaux dans le monde arabe, fait remarquer l'auteur, « durera tant que l'aspect progrès et émancipation de la Révolution française l'emportera sur le côté expansion et aventure étrangère " (p. 42).

Ces deux derniers articles, qui se situent à un niveau moins théorique et plus concret, convergent donc en ce qu'ils évoquent les modalités pratiques de l'impact des idéaux révolutionnaires sur les sociétés musulmanes : innovations, réformes... Il y est beaucoup question de la modernisation de l'État, des efforts déployés pour élaborer des constitutions, de la promotion de l'éducation et du journalisme, mais peu de critiques radicales de la religion et de la tradition. Malgré l'intérêt de ces différents développements, on regrette l'absence d'analyses conceptuelles plus fines portant, par exemple, sur les notions mêmes de "révolution ", de " liberté ", d' " égalité "... Comment ces notions sont-elles retraduites dans les contextes arabo-musulmans par les intellectuels réformistes? Quels sont les obstacles réels que rencontrent ces intellectuels? Poser ces questions nous aiderait peut-être à préciser les limites de l'universalisme des idéaux de la Révolution française.

Les trois derniers articles sont plus hétérogènes. Ils concernent respectivement un épisode de l'histoire du Japon, une caractéristique de l'idéologie républicaine en Amérique latine et un aspect de la pensée soviétique.

Kenji Kawano propose une nouvelle lecture d'un mouvement politique japonais de la fin du xIX ${ }^{\mathfrak{e}}$ siècle : « La Révolution française et Meiji Ishin " (p. 47-54). L'idée même d'établir ce parallélisme constitue une innovation dans l'historiographie japonaise, selon laquelle « les changements des années 1860 n'ont pas eu le caractère d'une révolution et n'ont pas entraîné la création d'un État moderne " (p. 47). L'auteur de l'article s'élève contre cette interprétation et, sans nier les différences qui séparent ces deux formidables bouleversements, en particulier du point de vue de la situation économique, met l'accent sur les convergences qui apparaissent dans le domaine politique et idéologique.

L'article de Luis Castro Leiva porte sur «Les paradoxes des révolutions hispano-américaines" (p. 55-69). Il expose les préoccupations des LatinoAméricains «quant à la validité et à l'applicabilité historique du concept de liberté » (p. 56) et montre comment ceux-ci ont été amenés à "mettre toute la force de l'éloquence au service de la quasi-totalité des grands principes d'une philosophie politique de l'histoire inspirée du Siècle des Lumières " (p. 58) avant d'analyser la corruption progressive par laquelle l'art oratoire cède la place, d'une part, au « textualisme constitutionnel ", d'autre part, à «l'éloquence des armes » (p. 62). En définitive, conclut-il, « la validité esthétique, éthique et politique de la philosophie des Lumières continue plus ou moins à régenter la conception intime que les Latino-Américains ont des passions et des actions politiques " (p. 66).

Enfin, Boris I. Koval présente « La morale humaniste de la Révolution française : une vision soviétique $»$ (p. 71-82). Cet auteur pense que les idées et les aspirations révolutionnaires, loin d'être tombées dans l'oubli, «continuent de nourrir les actes des hommes des générations actuelles, et avec plus de force encore peut-être qu'il y a deux cents ans " (p. 72). D'où la nécessité, en un temps 
où se précisent les menaces d'une guerre nucléaire et d'un anéantissement total de l'humanité, de ne pas s'en tenir à la portée politique et économique de la Révolution française, comme on le fait trop souvent, mais d'accorder l'intérêt le plus soutenu à sa dimension morale.

Concluons en exprimant l'espoir que l'initiative de la Revue internationale des sciences sociales stimulera les recherches historiques, sociologiques et philosophiques sur les influences et les interactions qui s'exercent d'une civilisation à une autre, d'un contexte culturel à un autre. Le cas de la Révolution française, eu égard à son exceptionnelle importance et à son irremplaçable rayonnement, constitue, bien entendu, un terrain privilégié qui, au-delà de l'actualité du Bicentenaire, ne doit jamais cesser d'être exploré. Mais des problématiques comparables ne peuvent-elles aussi être appliquées à d'autres cas ?

Ali Chenoufi.

Révolutions françaises et pensée allemande 1789/1871. Prés. par Lucien CaLví. Grenoble, Ellug, 1989. 14,5 × 20,5, 137 p. ("Centre d'études et de recherches sur les Allemagnes et l'Autriche contemporaines (C.E.R.A.A.C.)/Université Stendhal, Grenoble III »).

D'emblée s'impose au lecteur l'observation qu'il y a une distorsion entre l'ambition affichée par le titre et les dimensions modestes de l'ouvrage : comment couvrir en quelque cent quarante pages et en sept contributions le champ immense constitué par quatre révolutions et près de cent années d'histoire, au cours desquelles la référence, positive ou négative, à la Révolution de 1789 est une constante de la pensée allemande? Plus qu'en tout autre cas se pose ici la question du centrage thématique et chronologique, d'autant plus que l'étude qui ouvre le recueil se situe nettement en dehors des limites temporelles annoncées: Michel Espagne y étudie « la constitution d'une sociabilité maçonnique dans un cadre où se fondent les références françaises et allemandes " et publie en traduction française un traité sur l'éducation (retrouvé à la Bibliothèque nationale) rédigé avant 1789 par un franc-maçon allemand résidant à Paris. Le thème défini par le titre n'est vraiment abordé qu'avec la contribution de Jacques D'Hondt qui, à partir d'un détail peu signifiant à première vue, apporte un éclairage insolite et critique sur la relation de Hegel à la Révolution, en suivant à travers l'œuvre les avatars d'un bon mot réputé français, selon lequel « il n'est pas de héros pour son valet de chambre ". Les vues de Hegel à ce sujet sont révélatrices de certaines inconséquences de son système, de son peu d'intérêt pour l'histoire concrète et de ses préjugés en face de la situation réelle des couches plébéiennes. L'article de Jean Isler est de conception toute différente : il vise à donner une vue d'ensemble sur l'évolution d'un auteur, Görres, dont la réflexion politique s'étend de 1797 à 1848. Le thème de la révolution - celle de 1789 , dont il fut d'abord un ardent partisan dans la Rhénanie occupée, celle de 1830 , qu'il a observée, et celle de 
1848, qu'il a pressentie - est partout présent dans l'itinéraire de ce " renégat du bonnet phrygien " (Ernst Bloch), allant du cosmopolitisme révolutionnaire au nationalisme romantique conservateur. Cette évolution, on le sait, est représentative de celle de la jeune génération romantique qui va, pendant plus de trente ans, imprimer une orientation nouvelle à la culture allemande, jusqu'au moment où commencera à se restaurer dans les idéologies allemandes la référence positive à la Révolution française. C'est le cas avec Heine, auquel Norbert Biscons consacre une étude qui révèle l'importance - explicite et surtout implicite - de Montesquieu dans la genèse de sa pensée politique avant 1830 .

Les trois dernières contributions, qui portent sur Marx, constituent le centre réel du recueil. Lucien Calvié - dont on connaît par ailleurs l'édition des textes de Marx sur la Révolution française - retrace les diverses formes de l'idée de révolution dans le "Vormärz » : chez un " Jacobin », Ludwig Börne, un Jeune Hégélien, Eduard Gans, dans la Jeune Allemagne (Gutzkow) et chez Arnold Ruge, pour constater que c'est seulement chez Heine et surtout chez Marx que se dégage la conviction nouvelle qu'à la révolution de type français, impossible en Allemagne, doit se substituer une révolution d'un genre nouveau, sociale et prolétarienne. De la sorte se trouve à la fois maintenue et dépassée la référence à 1789-1793, dans le cadre d'un nouvel optimisme ouvert sur l'avenir. Une idée directrice analogue se retrouve dans les deux dernières contributions. Celle de Jacques Guilhaumou, linguiste (cf. son ouvrage La Langue politique et la Révolution française, Paris, Klincksieck, 1989), se penche sur la question du langage dans le marxisme naissant, plus précisément sur la traductibilité du langage politique jacobin dans la langue philosophique allemande, le problème étant de déterminer comment Marx a lu les mots de la Révolution dans la phase jacobine. Enfin, Christian Klein s'interroge sur la résurgence du débat entre deux principes opposés, le centralisme jacobin et le fédéralisme décentralisateur, dans l'analyse que donne Marx de la Commune de Paris dans La Guerre civile en France. Cette dernière étude confirme, dans le cadre de la référence restée incontournable à la première Révolution, la recherche par Marx de voies révolutionnaires nouvelles.

Une réunion d'études ponctuelles ne constitue certes pas une histoire, d'autant que le groupement présenté ici parait, sauf dans la seconde moitié de louvrage, quelque peu fortuit et arbitraire. Il va de soi que cette absence regrettable de centrage ne diminue en rien la qualité des contributions particulières. De même, on ne peut qu'approuver la méthode généralement suivie qui, évitant d'attribuer aux idèes et aux systèmes une dynamique autonome, les analyse « à l'intérieur des réalités sociales et du mouvement historique " ( $L$. Calvié, Présentation, p. 4). Il faut saluer ici un véritable renouvellement de l'ancienne "histoire des idées" dans la germanistique. 
" Révolte et société ", actes du IV colloque d'Histoire au présent, Paris, mai 1988, t. I et II, Sources. Travaux historiques, 15-16 et 17-18, 1988 et 1989.

C'était une gageure en ces temps de commémoration de la Révolution française : se pencher sur le mécanisme de la révolte qui ne peut être dans son acception la plus large que révolution avortée, et la disséquer pour mieux saisir ses relations avec la société d'où elle surgit et où elle retombe. Les quatre grandes parties de l'ouvrage soulignent la volonté des organisateurs de retrouver la signification de la révolte dans la longue durée en conjuguant les temps de l'histoire car il n'est pas interdit de trouver dans ces révoltes anciennes et contemporaines des modèles pour l'avenir.

La première partie " Révolte et légitimité " rassemble les articles qui traitent des fondements de la révolte ; car, sans légitimité implicite ou formulée, point de révolte, et, sans reconnaissance d'un droit de résister, point de soulèvement spontané ou organisé. Dans les sociétés anciennes, la révolte naît des empiètements de pouvoir sur un corps social tantôt sur la défensive, tantôt prêt à l'offensive. Ainsi, profondément antagonistes, bourgeois et paysans ont mené, chacun dans leur camp, un combat contre les seigneurs ou la monarchie. Leur échec ou leur réussite incomplète tient à l'impossibilité d'une solidarité. Est-ce pour la forger que l'on en vient à une codification de la révolte où l'idéologie sous-tend action et revendications? Plusieurs communications montrent comment l'époque contemporaine fournit maints exemples de passage "du pacte social à la codification de la révolte ".

Dès lors, le discours - appel, contenu, récit - joue un rôle primordial dans le déclenchement, le déroulement, la représentation et le retentissement des révoltes. Logiquement, la seconde partie de l'ouvrage regroupe les communications consacrées à la relation "Discours et Révolte " avec la dimension vécue et imaginaire des mouvements de révolte sans laquelle ils échapperaient au souvenir et à la transfiguration de la mémoire des hommes. Ici se rejoignent : " Jacqueries d'hier et d'aujourd'hui. "

Mais les ressemblances n'effacent ni les différences, ni les changements dans la nature des mouvements de révolte. C'est pourquoi les troisième et quatrième parties de l'ouvrage mettent en valeur les conséquences de l'évolution des sociétés sur le mécanisme et le déroulement des révoltes. Ainsi, l'âge des révoltes rurales et urbaines, caractéristiques en Europe des époques médiévale et moderne, durerait jusqu'au XIX ${ }^{\mathfrak{l}}$ siècle, offrant une permanence des acteurs et des formes d'action, ces " répertoires de la révolte "primitive"». Les changements survenus aux $X X^{e}$ et $X X^{e}$ siècles auraient pour origines l'élargissement des espaces et l'entrée en scène d'un autre modèle pour un autre monde : celui des révoltes coloniales dans les pays du Tiers-Monde. Jusqu'où va leur spécificité ? Telle est la question que se posent les auteurs des communications consacrées à ce thème. La dernière partie concerne la période contemporaine et fait la part belle à « Mai 68 ». Les "événements" y sont étudiés dans le prolongement des révoltes étudiantes, en fonction de l'éclosion de la jeunesse d'après-guerre et des « lectures " politique et syndicale qu'ils suggèrent. Cinq communications s'inscrivent, 
enfin, dans l'actualité en s'interrogeant sur " la révolte aujourd'hui : crise ou renouveau ".

Soulignons l'ampleur de l'enquête ainsi menée à bien jusqu'à nos jours et saluons la référence constante des auteurs à des exemples précis, afin de mieux saisir les mécanismes propres à chaque révolte et le fonds commun de ces affrontements de société. Même si, au premier regard, la singularité l'emporte et rend difficiles rapprochements et traits d'union entre les pays ou les siècles, il existe des constantes mises en valeur par les auteurs ou découvertes par le lecteur grâce à l'enchaînement chronologique et thématique de l'ouvrage. Dès lors, les plus connus de ces mouvements apparaissent sous un éclairage différent et reçoivent une interprétation nouvelle mais non définitive. Il suffit, en effet, de souligner le nombre de communications aux titres interrogatifs pour comprendre que l'intérêt des analyses proposées repose aussi dans les questions qu'elles suscitent. Comment le chantier de ces travaux pourrait-il ètre fermé puisque la révolte, dévoreuse insatiable, nourrit son originalité de la multiplicité des modèles antérieurs et des changements du corps social ? Saluons, enfin, les incitations à la réflexion contenues dans la préface de $M$. Vovelle, la conclusion de $R$. Rémond et dans les transitions habilement opérées par les maitres d'œuvre du colloque : Gh. Brunel, F. Gambrelle, F. Georgi, Ph. Jansen, F-O. Touati, M. Trebitsch. Reste le regret de ne pouvoir citer les noms des soixante-dix participants, auteurs des communications qui composent ces deux ouvrages. Mais il est atténué par le plaisir que le lecteur prendra en allant à leur rencontre.

Anne-Marie Cocul.A. 